\title{
Voxelwise meta-analysis of gray matter anomalies in progressive supranuclear palsy and Parkinson's disease using anatomic likelihood estimation
}

\author{
Na Shao, Jing Yang, Jianpeng Li and Hui-Fang Shang*
}

Department of Neurology, West China Hospital, Sichuan University, Chengdu, China

Edited by:

Silvio lonta, Swiss Federal Institute

of Technology Zurich (ETHZ),

Switzerland

Reviewed by:

Gerard R. Ridgway, University

College London, UK

Katja Franke, University Hospital

Jena, Germany

*Correspondence:

Hui-Fang Shang, Department of

Neurology, West China Hospital,

Sichuan University, Cheng Du Wai

Nan Guo Xue Xiang 37\#, 610041

Chengdu, China

e-mail: hfshang2002@126.com
Numerous voxel-based morphometry (VBM) studies on gray matter (GM) of patients with progressive supranuclear palsy (PSP) and Parkinson's disease (PD) have been conducted separately. Identifying the different neuroanatomical changes in GM resulting from PSP and PD through meta-analysis will aid the differential diagnosis of PSP and PD. In this study, a systematic review of VBM studies of patients with PSP and PD relative to healthy control $(\mathrm{HC})$ in the Embase and PubMed databases from January 1995 to April 2013 was conducted. The anatomical distribution of the coordinates of GM differences was meta-analyzed using anatomical likelihood estimation. Separate maps of GM changes were constructed and subtraction meta-analysis was performed to explore the differences in GM abnormalities between PSP and PD. Nine PSP studies and 24 PD studies were included. GM reductions were present in the bilateral thalamus, basal ganglia, midbrain, insular cortex and inferior frontal gyrus, and left precentral gyrus and anterior cingulate gyrus in PSP. Atrophy of GM was concentrated in the bilateral middle and inferior frontal gyrus, precuneus, left precentral gyrus, middle temporal gyrus, right superior parietal lobule, and right cuneus in PD. Subtraction meta-analysis indicated that GM volume was lesser in the bilateral midbrain, thalamus, and insula in PSP compared with that in PD. Our meta-analysis indicated that PSP and PD shared a similar distribution of neuroanatomical changes in the frontal lobe, including inferior frontal gyrus and precentral gyrus, and that atrophy of the midbrain, thalamus, and insula are neuroanatomical markers for differentiating PSP from PD.

Keywords: progressive supranuclear palsy, Parkinson's disease, subtraction meta-analysis, anatomic likelihood estimation, voxel-based morphometry, gray matter volume

\section{INTRODUCTION}

Progressive supranuclear palsy (PSP) is an atypical Parkinsonism neurodegenerative disease that often affects adults between 60 and 65 years of age (Armstrong, 2007). PSP is characterized by progressive vertical gaze palsy, early postural instability with falls, late frontal cognitive dysfunction, and poor response to levodopa (Barsottini et al., 2010; Hirano et al., 2010; Stamelou et al., 2010). Recent studies have suggested that the morbidity of PSP may be higher than is usually considered (Nath et al., 2001). Parkinson's disease (PD), the second most common neurodegenerative disease, is characterized by rigidity, tremor, bradykinesia, and posture instability (Tanner and Goldman, 1996). Although symptoms such as vertical gaze palsy and early falls are specific to PSP, differential diagnosis from PD can still be difficult at early stages (Nilsson et al., 2007; Varrone et al., 2007).

Structural magnetic resonance imaging (MRI), functional MRI (fMRI), and positron emission tomography (PET) are increasingly being used in the differential diagnosis of $\mathrm{PD}$ and related disorders (Juh et al., 2004). Voxel-based morphometry (VBM), a time-efficient and automated tool, can reveal morphological changes throughout the whole brain on a voxel-wise basis (Ashburner and Friston, 2000). VBM has been widely used in studies on PSP and PD (Brenneis et al., 2004; Burton et al., 2004; Beyer et al., 2007; Agosta et al., 2010). However, the results of VBM studies are controversial or not completely consistent because of small and heterogeneous samples of participants and substantial methodological differences among studies. For example, some researchers (Agosta et al., 2010; Lehericy et al., 2010) have reported cerebellum atrophy in PSP, whereas others (Brenneis et al., 2004; Boxer et al., 2006) did not find such abnormalities in PSP. Similarly, some articles (Focke et al., 2011; Fernández-Seara et al., 2012) have reported precuneus impairment in PD, whereas others (Dalaker et al., 2010; Hong et al., 2012) failed to replicate such findings in PD. Thus, the application of meta-analysis to identify consistent results of VBM studies on PSP and PD has gained increasing interest. We published a VBM meta-analysis on PD in 2012, which included articles from 1995 to 25 October 2010 (Pan et al., 2012). As more VBM studies on PD published since then, we updated the results of meta-analysis on PD to explore more accurate GM changes in PD.

The anatomic likelihood estimate (ALE) method, a newly developed statistical technique, is a powerful voxel-based metaanalytic technique originally designed for functional neuroimaging studies (Turkeltaub et al., 2002; Laird et al., 2005). However, ALE has been effectively applied to anatomical 
image datasets, such as VBM and diffusion tensor imaging studies (Ellison-Wright et al., 2008; Ellison-Wright and Bullmore, 2009). Identifying consistent structural abnormalities may provide insight into the pathophysiology of PSP and PD. PSP can be easily misdiagnosed as PD. Given that the midbrain and thalamus are reportedly more severely damaged in PSP, and that the impairment of these two areas contributes to classical symptoms, such as vertical gaze palsy (Kato et al., 2003) and postural instability (Zwergal et al., 2011), we speculated that atrophy of the midbrain and thalamus may be a neuroanatomical marker for the differentiation of PSP from PD. Therefore, subtracting the results of metaanalysis of PSP and PD will reveal the difference in pattern change between PD and PSP, thus confirming our abovementioned hypothesis and increasing diagnostic accuracy in clinical practice.

This study aimed to identify the consistent finding of gray matter volume (GMV) changes in PSP and PD separately using the ALE approach (Turkeltaub et al., 2002; Laird et al., 2005). Contrast analysis was performed through ALE subtraction analysis (Eickhoff et al., 2011) to identify the differential pattern of GMV changes between PSP and PD.

\section{MATERIALS AND METHODS \\ DATA SOURCES AND STUDY SELECTION}

Systematic and comprehensive searches were conducted on the Embase and PubMed databases from January 1995 to April 2013 using the following key words: ("voxel," "voxelbased," "voxel-wise," "morphometry," "VBM,") and ("PSP," "Steele-Richardson-Olszewski syndrome," "ophthalmoplegia," "Parkinson," "Parkinsonism," "Paralysis Agitans," and "PD"). The reference lists of relevant papers were also searched for additional studies.

Studies were included if they met the following criteria: (1) reported on VBM as applied to GM density (GMD) or GMV changes from a comparison of PSP and healthy control (HC), or comparison of PD and HC (PD-HC), or comparison of PSP and PD (PSP-PD); (2) reported whole-brain GM changes in standard Talairach or Montreal Neurological Institute (MNI) stereotactic spatial coordinates; (3) used significance thresholds that are either corrected for multiple comparisons or uncorrected but with spatial extent thresholds; (4) were published in English with peer review. For studies that met the aforementioned inclusion criteria and were performed by the same authors or research institution, only the study with the largest sample size was selected.

Studies were excluded if they suffered from at least one of the following deficiencies: (1) sufficient data could not be obtained even after corresponding with author/s by email or phone; (2) the data overlapped with those of another article.

The method used in the current study was based on the guidelines of "Meta-analysis of Observational Studies in Epidemiology" for the meta-analysis of observational studies (Stroup et al., 2000).

Study selection was performed by one author (Na Shao) and verified by another author (Jing Yang) independently. The process of study selection was provided in Figure 1.

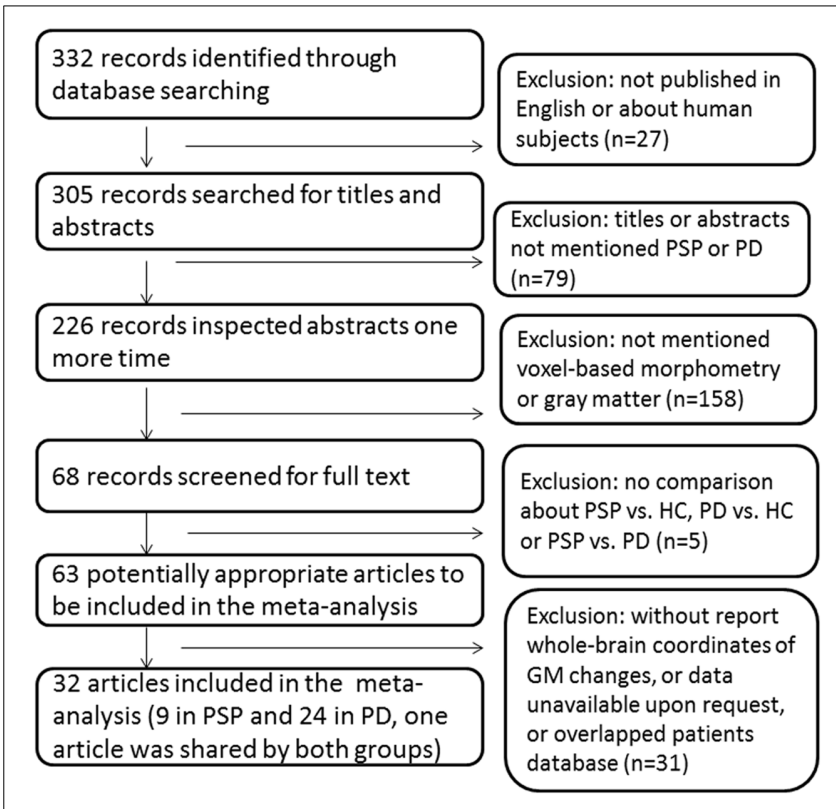

FIGURE 1 | Flow-chart of searching strategy.

\section{DATA EXTRACTION}

Ginger ALE (http://brainmap.org/ale/index.html) was used to transform reported coordinates into the stereotactic space of Talairach. Reported coordinates in a stereotactic space of the MNI were converted to Talairach coordinates using Lancaster transform (Lancaster et al., 2007). Reported coordinates transformed to Talairach space using the Brett transformation (Brett et al., 2002) were converted back to MNI space and then reconverted to Talairach also using the Lancaster formula (Lancaster et al., 2007). Two authors (Na Shao and Jing Yang) performed data extraction independently.

\section{META-ANALYSIS OF VBM STUDIES}

Meta-analysis was performed using Ginger ALE software 2.1.1 (http://www.brainmap.org) and the Talairach stereotactic coordinates derived from the included articles (Eickhoff et al., 2012). The reported coordinates were modeled using a 3D Gaussian distribution defined by full width at half maximum (FWHM) (Laird et al., 2005). The FWHM was set according to a quantitative uncertainty model (Eickhoff et al., 2009). Separate ALE maps were created for multiple comparisons associated with PSP-HC and PD-HC. Afterward, in order to identify the differential pattern of GMV changes between PSP and PD, ALE subtraction analysis (Eickhoff et al., 2011) evaluating the differences between the ALE maps of PSP-HC and PD-HC was carried out, taking into account the potential difference in the sample size. These maps were thresholded at $p<0.05$ corrected for multiple comparisons using False Discovery Rate (Benjamini and Hochberg, 1995). An extent-threshold of $100 \mathrm{~mm}^{3}$ was applied. The coordinates of the weighted center were generated for each cluster. The resulting significant anatomical areas were labeled by referring to probabilistic cytoarchitectonic maps of the human brain using the SPM Anatomy Toolbox v1.8 (Eickhoff 
et al., 2005). This labeling presented the attribution of each voxel of the reference space to the most likely cytoarchitectonic area (Eickhoff et al., 2005). Rest software was used to visualize the ALE maps overlaid onto an average brain template MNI/ICBM 152 (http://www.bic.mni.mcgill.ca/ServicesAtlases/ ICBM152NLin2009).

\section{RESULTS}

\section{PSP $<$ HC}

We identified 32 studies in the database searches (Figure 1) including nine PSP-HC articles (Brenneis et al., 2004; Cordato et al., 2005; Boxer et al., 2006; Padovani et al., 2006; Agosta et al., 2010; Lehericy et al., 2010; Takahashi et al., 2011; Ghosh et al., 2012; Giordano et al., 2013). One hundred and fourty-five PSP patients and $217 \mathrm{HC}$ were included in this study and a total of 104 foci with reduced GMV were reported. The main characteristics of the included participants are presented in Table 1. Thirty PSP patients from two studies had mild cognitive impairment (Padovani et al., 2006; Takahashi et al., 2011). GM reduction was observed in the bilateral thalamus, midbrain, insula, basal ganglia, inferior frontal gyrus, left precentral gyrus, and the left anterior cingulate by using the ALE approach (Figure 2). No increased GMV relative to $\mathrm{HC}$ was observed in the brain regions of PSP patients. Similar findings were revealed after excluding two studies conducted on PSP patients with mild cognitive impairment (Padovani et al., 2006; Takahashi et al., 2011) (Supplemental Figure 1).

\section{PD $<$ HC}

Thirty-two studies were identified in the meta-analysis (Figure 1) and a total of 24 studies on PD were included (Burton et al., 2004; Cordato et al., 2005; Nagano-Saito et al., 2005; Beyer et al., 2007; Ramirez-Ruiz et al., 2007; Feldmann et al., 2008; Karagulle Kendi et al., 2008; Camicioli et al., 2009; Jubault et al., 2009; Martin et al., 2009; Pereira et al., 2009; Sanchez-Castaneda et al., 2009; Tir et al., 2009; Dalaker et al., 2010; Kostic et al., 2010; Lee et al., 2010; Cerasa et al., 2011; Focke et al., 2011; Meppelink et al., 2011; Compta et al., 2012; Fernández-Seara et al., 2012; Hong et al., 2012; Ibarretxe-Bilbao et al., 2012; Tessitore et al., 2012). Seven hundred and sixteen PD patients and $535 \mathrm{HC}$ subjects met the inclusion criteria for meta-analysis. The main characteristics of these included populations are presented in Table 2. Among these participants, 60 patients from three studies had mild cognitive impairment (Beyer et al., 2007; Dalaker et al., 2010; Lee et al., 2010) and 16 patients from one study had dementia (Sanchez-Castaneda et al., 2009). A total of 117 foci presented with decreased GMV were reported. ALE metaanalysis identified reduced GMV in the following: (1) frontal lobe including the bilateral middle, inferior frontal gyrus, and left precentral gyrus, (2) parietal lobe including bilateral precuneus and right superior parietal lobule, and (3) left middle temporal gyrus and right cuneus (Figure 2). No increased regions relative to $\mathrm{HC}$ were found in $\mathrm{PD}$ patients. Similar findings were obtained after excluding the aforementioned four studies conducted on PD patients with cognitive impairment (Supplemental Figure 1).

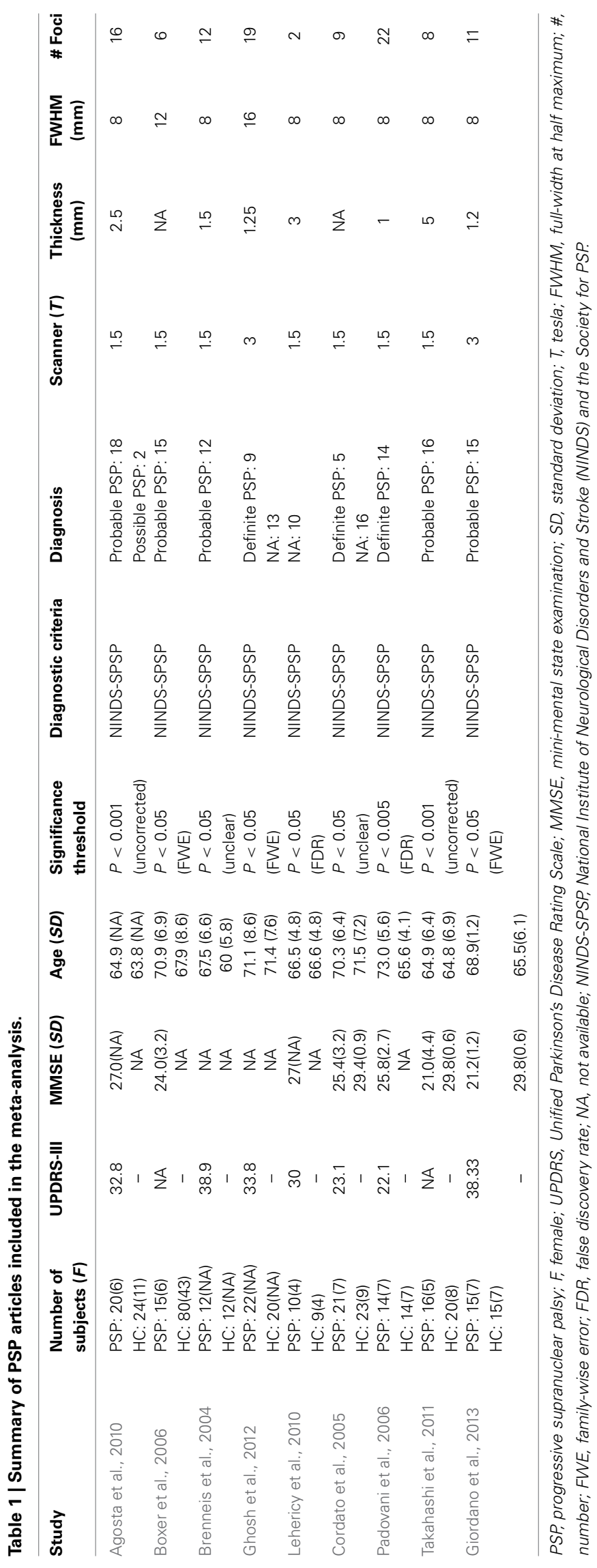




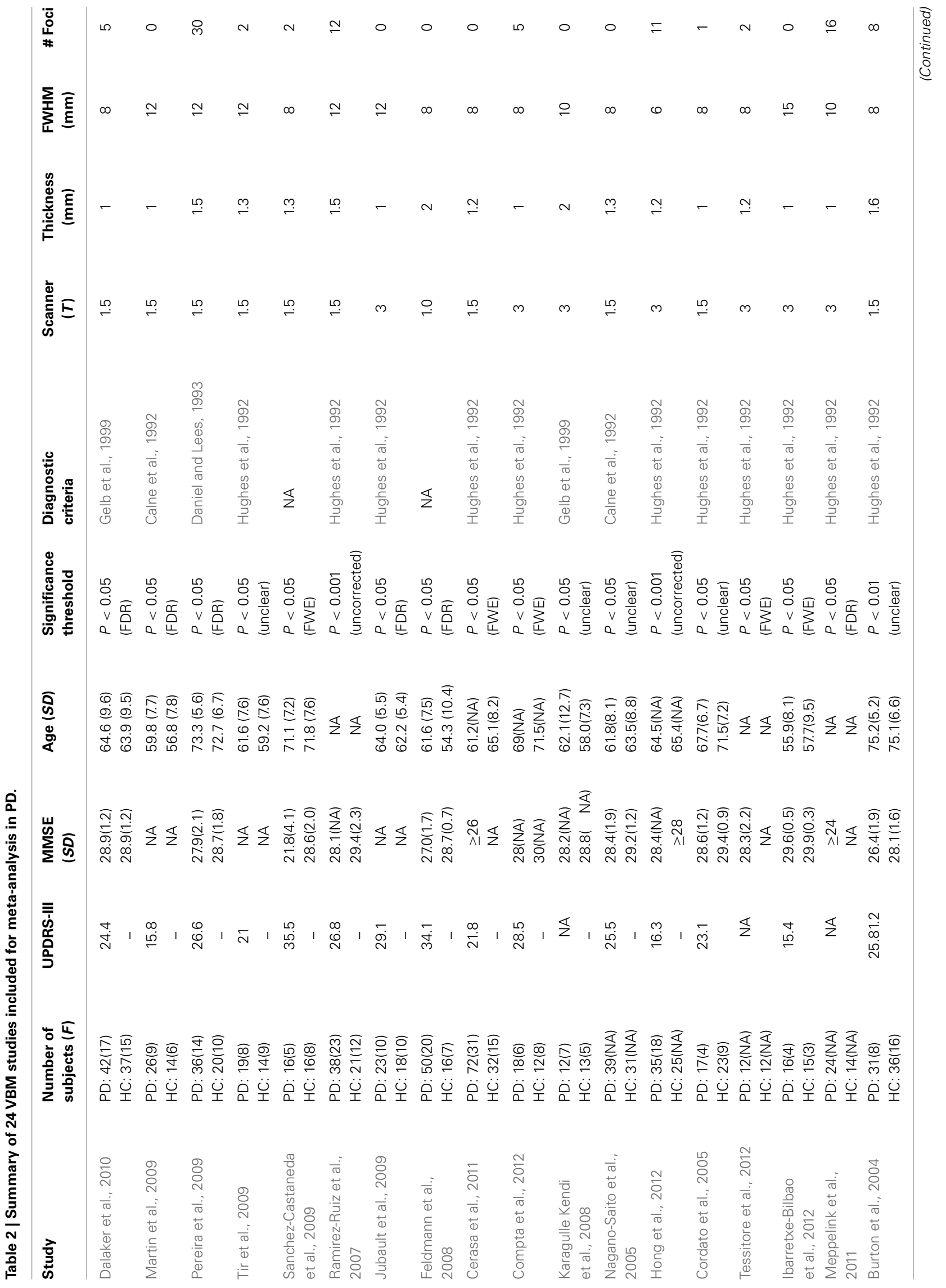




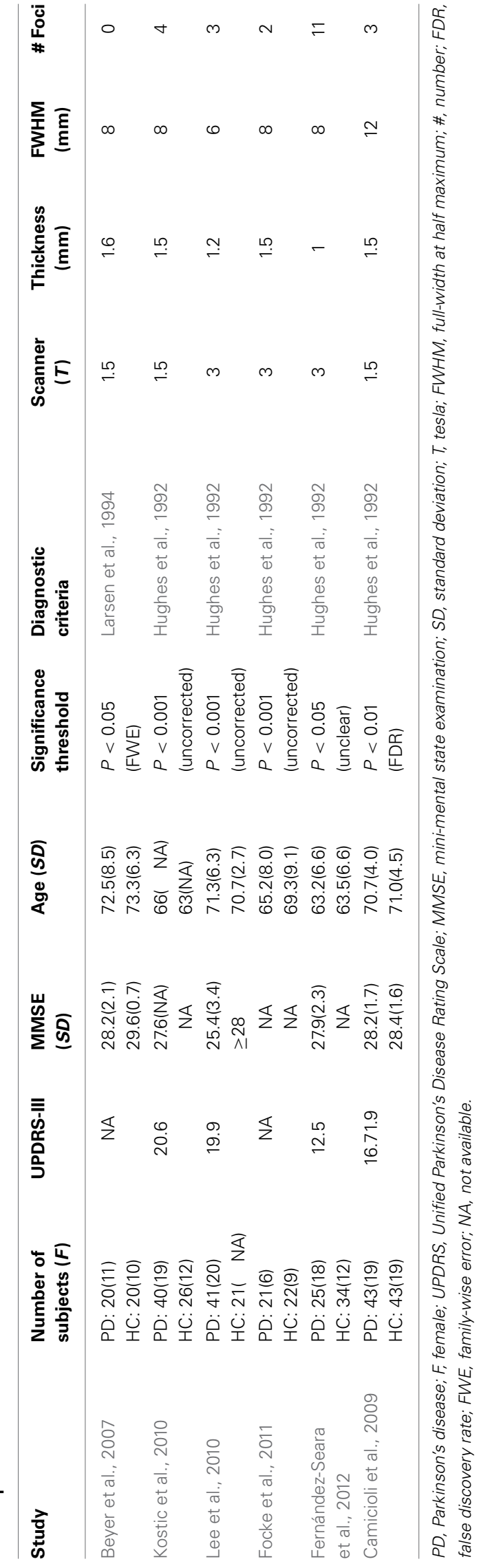

\section{PSP $<$ PD}

Given that only two of three studies (Cordato et al., 2005; Focke et al., 2011; Giordano et al., 2013) directly investigated GMV differences between PSP and PD using the whole brain coordinate (Focke et al., 2011; Giordano et al., 2013), meta-analysis was not highly reliable. Therefore, ALE subtraction analysis was performed to compare GM differences between PSP and PD (Eickhoff et al., 2011). The subtraction analysis identified decreased GMV in the bilateral midbrain, thalamus, and insula in PSP compared with PD (Table 3 and Figure 2). The reverse contrast did not show any regions of GM atrophy. Similar findings were found using subtraction analysis between PSP (non-MCI) and PD (non-MCI) studies without including patients with mild cognitive impairment or dementia (Supplemental Figure 1).

\section{DISCUSSION}

Quantitative meta-analysis of VBM studies on PSP showed decreased GMV in the thalamus, midbrain, basal ganglia, insula, and frontal lobe, whereas quantitative meta-analysis of VBM studies on PD revealed reduced GMV in the frontal lobe, parietal lobe, left middle temporal gyrus, and right cuneus. In addition, subtraction analysis showed GM atrophy in the thalamus, midbrain, and insula lobe in PSP compared with PD.

In this study, reduced GMV in PD was observed in the following: (1) frontal lobe including bilateral middle, inferior frontal gyrus, and left precentral gyrus, (2) parietal lobe including bilateral precuneus and right superior parietal lobule, and (3) left middle temporal gyrus and right cuneus. The previous results showed changes in PD localized in the left inferior frontal gyrus, left superior temporal gyrus, and left insula lobe (Pan et al., 2012). The main results are consistent. The differences between the present findings and those of previous VBM studies on PD may be the result of the difference in methods ("signed differential mapping" method was used in our previous study and ALE method was used in the present study) and number of participants (498 PD patients and $375 \mathrm{HC}$ were included in our previous study, whereas $716 \mathrm{PD}$ patients and $535 \mathrm{HC}$ were included in the present study).

We found decreased GMV in the frontal lobe, including inferior frontal gyrus and precentral gyrus in both PSP and PD. These results are supported by some fMRI and pathological studies (Albers et al., 2000; Whitwell et al., 2011; Kehagia et al., 2012; Yang et al., 2013). The precentral gyrus belongs to the motor cortex and is the origin of the pyramidal tract (Bonelli and Cummings, 2007). Additionally, the inferior frontal gyrus (Brodmann area 44), affects movement control, including speech and hand actions (Rizzolatti et al., 2002; Bonelli and Cummings, 2007). It also participates in response inhibition (Picton et al., 2007; Forstmann et al., 2008). Therefore, the impairment of the precentral gyrus and inferior frontal gyrus may partially contribute to the motor and non-motor deficits of both disorders.

Interestingly, GM atrophy in the bilateral midbrain, thalamus, and insula was found to be more severe in PSP than in PD, although no significant foci were revealed in these structures in the meta-analysis of VBM studies on PD. The pathological hallmarks of PSP include globose neurofibrillary tangles, tufted taupositive astrocytes, and neuronal loss in the midbrain (Dickson 


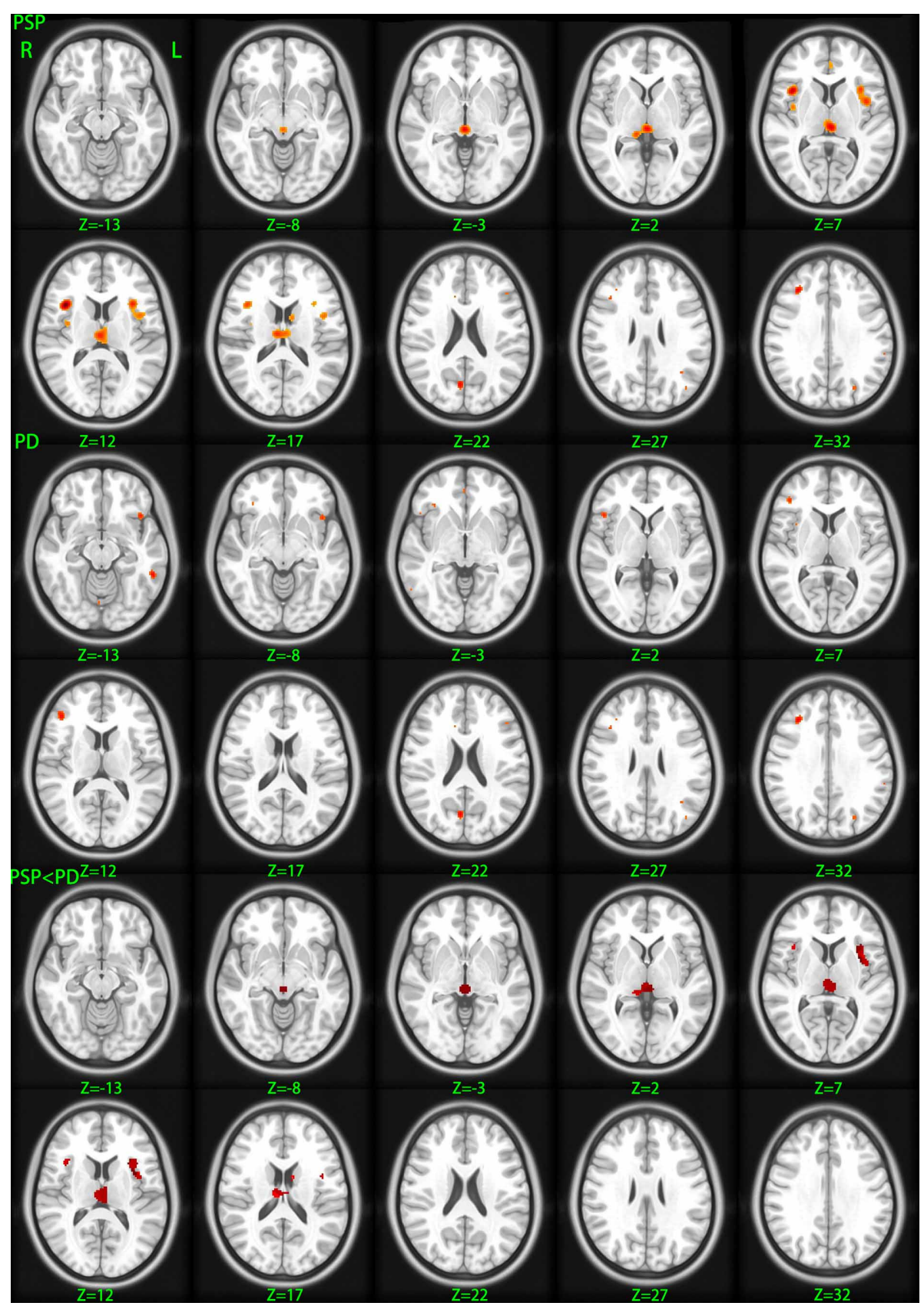

FIGURE 2 | Gray matter volume changes betweeen different groups. PSP, gray matter decreases in patients with progressive supranuclear palsy relative to healthy control; $P D$, gray matter decreases in patients with Parkinson's disease relative to healthy control; PSP $<$ PD, diminished gray matter volume in progressive supranuclear palsy patients relative to Parkinson's disease patients. Significance thresholded with a false discovery rate at $P<0.05$ and a cluster extent threshold of 100 voxels; $z, z$ coordinates in Talairach space; $L$, left; $R$, right. et al., 2007; Williams and Lees, 2009). The midbrain not only maintains equilibrium (Barsottini et al., 2010), but also has a role in vertical saccades (Da Cunha et al., 2002; Kato et al., 2003). More severe atrophy of the midbrain can contribute to disturbances in balance and vertical gaze movement in PSP. Although dopaminergic neuron loss in the substantia nigra in $\mathrm{PD}$ was found to be the main pathology in PD (Halliday et al., 1996), we failed to find midbrain atrophy in PD by means of VBM meta-analysis. Therefore, severe atrophy of the midbrain could be a neuroanatomical marker that can be used to differentiate PSP from PD.

Subtraction analysis showed that GMV reductions in the bilateral thalamus are more severe in PSP patients compared with PD patients. Atrophy of the thalamus revealed in PSP in the current meta-analysis of VBM studies is consistent with the findings of studies using PET (Juh et al., 2005) and 
Table 3 | Changes in gray matter volume.

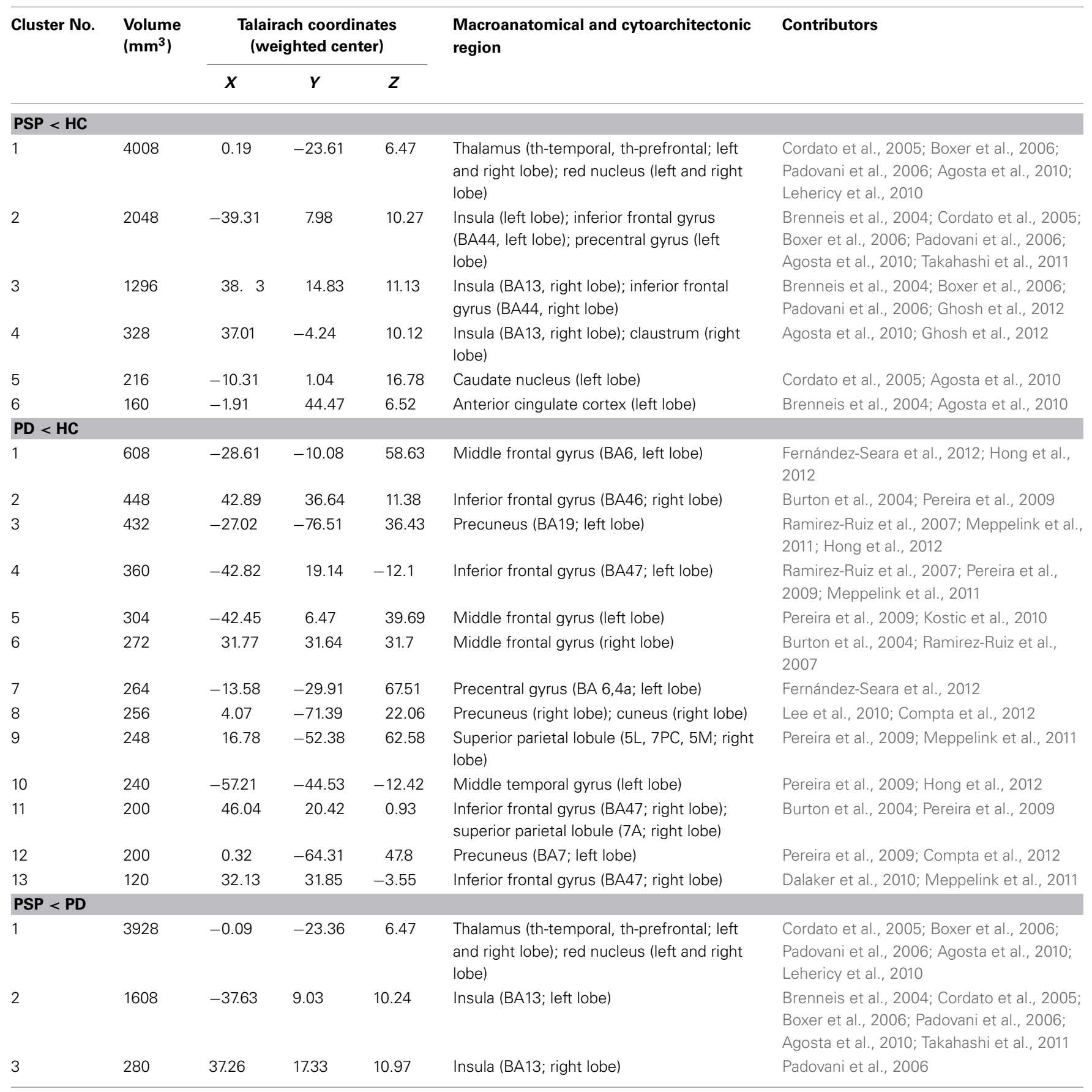

No., number; PSP, progressive supranuclear palsy; HC, healthy control; PD, Parkinson's disease; th-, thalamus; BA, Brodmann area.

single-photon emission computed tomography (Kimura et al., 2011). Pathological research on PSP also confirmed that the thalamus often exhibits some degree of neuronal loss and gliosis, particularly in the lateral and ventral anterior thalamus nuclei (Dickson et al., 2007). Postural instability in PSP patients has been associated with impairment of the thalamus (Zwergal et al., 2011), which has a significant role in motor functions through both functional and structural connections with the basal ganglia and motor regions of the frontal lobe (Herrero et al., 2002; Cordato et al., 2005; Bonelli and Cummings, 2007). Although altered thalamic neuronal activity in the cortical-striatal-thalamic loop is the main pathophysiology in PD (Halliday, 2009), the present study found no obvious thalamus atrophy in PD, confirming that thalamus impairment in PD consists of functional deficits other than structural changes. Our main findings stayed consistent after excluding PSP or PD patients with cognitive impairment, we can 
not, suggest that the thalamus may not be closely related to the cognitive impairment of PD or PSP patients since these PSP or PD patients with cognitive impairment accounted for a small portion of our patient samples. However, future studies on PSP or more advanced PD patients will increase current understanding on the impairment of the thalamus.

Interestingly, subtracting analysis showed that insula atrophy was more severe in PSP than in PD. Very few articles have investigated the insula impairment of PSP. A previous PET study found hypometabolism in the insula of PSP patients (Hirano et al., 2010). Other studies have found that dysfunction of the insula could lead to abnormalities in articulation and emotions (Augustine, 1996; Baldo et al., 2011). Although some neuroimaging studies using structural MRI, fMRI, or PET (Kikuchi et al., 2001; Stefurak et al., 2003; Pinto et al., 2004; Pavese et al., 2010) have reported insula damage in PD patients, these patients exhibited fatigue or dysarthria. Most included studies on PD did not provide detailed information on motor and non-motor symptoms, such as severity of dysarthria, static tremor, or fatigue, which may partially explain why insula atrophy was not proved in this study.

However, the finding of subtraction analysis in the current study was different from the results of two VBM studies (Focke et al., 2011; Giordano et al., 2013) which directly compared GMV on PSP and PD, which found the atrophy was more severe in cerebellum, parahippocampal gyrus, middle frontal gyrus, occipital pole, and precentral gyrus in PSP than PD. However, the sample size of these two studies was relatively small. Future large sample size comparison studies on PSP-PD with multimodal imaging techniques will help to answer the difference. This study has several limitations, which could have biased our results. First, unpublished studies and studies with insufficient data were excluded, and the studies were limited to those published in English. Second, the voxel-wise meta-analyses were based on the reported stereotactic coordinates with significant differences rather than on raw data, and the included patients from different studies may have experienced various severities of disease or therapy, thereby potentially influencing the results. Third, the heterogeneity of the methodologies used in VBM studies, including different expression of GM changes (modulated GMV and unmodulated GMD), FWHM and statistical thresholding methods, could not be ruled out entirely. Additionally, PSP is classified into Richardson syndrome and PSP-Parkinsonism (PSP-P) according to different clinical signs (Williams et al., 2005). Given that only two articles (Agosta et al., 2010; Lehericy et al., 2010) mention the phenotypes, we could not perform separate meta-analysis of each phenotype or between PSP-P and PD.

In summary, the present meta-analysis indicated that both PSP and PD patients suffer from GM atrophy in the precentral gyrus and inferior frontal gyrus. GM atrophy was also identified in the bilateral midbrain, thalamus, and insula in PSP patients compared with PD patients, and could thus be an anatomical biomarker that can be used to differentiate these two disorders. Future studies with larger samples of patients in the early stage of disease, as well as multimodal methods, may better expound the changes.

\section{ACKNOWLEDGMENTS}

We thank all the authors of the included studies.

\section{FUNDING}

We had no funding in this study.

\section{SUPPLEMENTARY MATERIAL}

The Supplementary Material for this article can be found online at: http://www.frontiersin.org/journal/10.3389/fnhum. 2014.00063/abstract

\section{REFERENCES}

Agosta, F., Kostic, V. S., Galantucci, S., Mesaros, S., Svetel, M., Pagani, E., et al (2010). The in vivo distribution of brain tissue loss in Richardson's syndrome and PSP-parkinsonism: a VBM-DARTEL study. Eur. J. Neurosci. 32, 640-647. doi: 10.1111/j.1460-9568.2010.07304.x

Albers, D. S., Augood, S. J., Park, L. C., Browne, S. E., Martin, D. M., Adamson, J., et al. (2000). Frontal lobe dysfunction in progressive supranuclear palsy: evidence for oxidative stress and mitochondrial impairment. J. Neurochem. 74, 878-881. doi: 10.1046/j.1471-4159.2000.740878.x

Armstrong, R. A. (2007). Progressive supranuclear palsy (PSP): general pathology and visual signs and symptoms. Optom. Today 2007, 48-52.

Ashburner, J., and Friston, K. J. (2000). Voxel-based morphometry-the methods. Neuroimage 11, 805-821. doi: 10.1006/nimg.2000.0582

Augustine, J. R. (1996). Circuitry and functional aspects of the insular lobe in primates including humans. Brain Res. Brain Res. Rev. 22, 229-244. doi: 10.1016/S0165-0173(96)00011-2

Baldo, J. V., Wilkins, D. P., Ogar, J., Willock, S., and Dronkers, N. F. (2011). Role of the precentral gyrus of the insula in complex articulation. Cortex 47, 800-807. doi: 10.1016/j.cortex.2010.07.001

Barsottini, O. G., Felicio, A. C., Aquino, C. C., and Pedroso, J. L. (2010). Progressive supranuclear palsy: new concepts. Arq. Neuropsiquiatr. 68, 938-946. doi: 10.1590/S0004-282X2010000600020

Benjamini, Y., and Hochberg, Y. (1995). Controlling the false discovery rate: a practical and powerful approach to multiple testing. J. R. Statistic. Soc. B (Methodological) 57, 289-300.

Beyer, M. K., Janvin, C. C., Larsen, J. P., and Aarsland, D. (2007). A magnetic resonance imaging study of patients with Parkinson's disease with mild cognitive impairment and dementia using voxel-based morphometry. J. Neurol. Neurosurg. Psychiatry 78, 254-259. doi: 10.1136/jnnp.2006.093849

Bonelli, R. M., and Cummings, J. L. (2007). Frontal-subcortical circuitry and behavior. Dialogues Clin. Neurosci. 9, 141-151.

Boxer, A. L., Geschwind, M. D., Belfor, N., Gorno-Tempini, M. L., Schauer, G. F., Miller, B. L., et al. (2006). Patterns of brain atrophy that differentiate corticobasal degeneration syndrome from progressive supranuclear palsy. Arch. Neurol. 63, 81-86. doi: 10.1001/archneur.63.1.81

Brenneis, C., Seppi, K., Schocke, M., Benke, T., Wenning, G. K., and Poewe, W. (2004). Voxel based morphometry reveals a distinct pattern of frontal atrophy in progressive supranuclear palsy. J. Neurol. Neurosurg. Psychiatry 75, 246-249. doi: 10.1136/jnnp.2003.015297

Brett, M., Johnsrude, I. S., and Owen, A. M. (2002). The problem of functional localization in the human brain. Nat. Rev. Neurosci. 3, 243-249. doi: $10.1038 / \mathrm{nrn} 756$

Burton, E. J., Mckeith, I. G., Burn, D. J., Williams, E. D., and O’brien, J. T. (2004). Cerebral atrophy in Parkinson's disease with and without dementia: a comparison with Alzheimer's disease, dementia with Lewy bodies and controls. Brain 127, 791-800. doi: 10.1093/brain/awh088

Camicioli, R., Gee, M., Bouchard, T. P., Fisher, N. J., Hanstock, C. C., Emery, D. J., et al. (2009). Voxel-based morphometry reveals extra-nigral atrophy patterns associated with dopamine refractory cognitive and motor impairment in parkinsonism. Parkinsonism Relat. Disord. 15, 187-195. doi: 10.1016/j.parkreldis.2008.05.002

Calne, D. B., Snow, B. J., and Lee, C. (1992). Criteria for diagnosing Parkinson's disease. Ann. Neurol. 32, S125-S127. doi: 10.1002/ana.410320721

Cerasa, A., Messina, D., Pugliese, P., Morelli, M., Lanza, P., Salsone, M., et al. (2011). Increased prefrontal volume in PD with levodopa-induced dyskinesias: a voxelbased morphometry study. Mov. Disord. 26, 807-812. doi: 10.1002/mds.23660 
Compta, Y., Ibarretxe-Bilbao, N., Pereira, J. B., Junque, C., Bargallo, N., Tolosa, E., et al. (2012). Grey matter volume correlates of cerebrospinal markers of Alzheimer-pathology in Parkinson's disease and related dementia. Parkinsonism Relat. Disord. 18, 941-947. doi: 10.1016/j.parkreldis.2012. 04.028

Cordato, N. J., Duggins, A. J., Halliday, G. M., Morris, J. G., and Pantelis, C. (2005). Clinical deficits correlate with regional cerebral atrophy in progressive supranuclear palsy. Brain 128, 1259-1266. doi: 10.1093/brain/awh508

Da Cunha, C., Angelucci, M. E., Canteras, N. S., Wonnacott, S., and Takahashi, R. N. (2002). The lesion of the rat substantia nigra pars compacta dopaminergic neurons as a model for Parkinson's disease memory disabilities. Cell. Mol. Neurobiol. 22, 227-237. doi: 10.1023/A:1020736131907

Dalaker, T. O., Zivadinov, R., Larsen, J. P., Beyer, M. K., Cox, J. L., Alves, G., et al. (2010). Gray matter correlations of cognition in incident Parkinson's disease. Mov. Disord. 25, 629-633. doi: 10.1002/mds.22867

Daniel, S. E., and Lees, A. J. (1993). Parkinson's Disease Society Brain Bank, London: overview and research. J. Neural. Transm. Suppl. 39, 165-172.

Dickson, D. W., Rademakers, R., and Hutton, M. L. (2007). Progressive supranuclear palsy: pathology and genetics. Brain Pathol. 17, 74-82. doi: 10.1111/j.17503639.2007.00054.x

Eickhoff, S. B., Bzdok, D., Laird, A. R., Kurth, F., and Fox, P. T. (2012). Activation likelihood estimation meta-analysis revisited. Neuroimage 59, 2349-2361. doi: 10.1016/j.neuroimage.2011.09.017

Eickhoff, S. B., Bzdok, D., Laird, A. R., Roski, C., Caspers, S., Zilles, K., et al. (2011). Co-activation patterns distinguish cortical modules, their connectivity and functional differentiation. Neuroimage 57, 938-949. doi: 10.1016/j.neuroimage.2011.05.021

Eickhoff, S. B., Laird, A. R., Grefkes, C., Wang, L. E., Zilles, K., and Fox, P. T. (2009). Coordinate-based activation likelihood estimation meta-analysis of neuroimaging data: a random-effects approach based on empirical estimates of spatial uncertainty. Hum. Brain Mapp. 30, 2907-2926. doi: 10.1002/hbm.20718

Eickhoff, S. B., Stephan, K. E., Mohlberg, H., Grefkes, C., Fink, G. R., Amunts, K., et al. (2005). A new SPM toolbox for combining probabilistic cytoarchitectonic maps and functional imaging data. Neuroimage 25, 1325-1335. doi: 10.1016/j.neuroimage.2004.12.034

Ellison-Wright, I., and Bullmore, E. (2009). Meta-analysis of diffusion tensor imaging studies in schizophrenia. Schizophr. Res. 108, 3-10. doi: 10.1016/j.schres.2008.11.021

Ellison-Wright, I., Glahn, D. C., Laird, A. R., Thelen, S. M., and Bullmore, E. (2008). The anatomy of first-episode and chronic schizophrenia: an anatomical likelihood estimation meta-analysis. Am. J. Psychiatry 165, 1015-1023. doi: 10.1176/appi.ajp.2008.07101562

Feldmann, A., Illes, Z., Kosztolanyi, P., Illes, E., Mike, A., Kover, F., et al. (2008). Morphometric changes of gray matter in Parkinson's disease with depression: a voxel-based morphometry study. Mov. Disord. 23, 42-46. doi: $10.1002 / \mathrm{mds} .21765$

Fernández-Seara, M. A., Mengual, E., Vidorreta, M., Aznarez-Sanado, M., Loayza, F. R., Villagra, F., et al. (2012). Cortical hypoperfusion in Parkinson's disease assessed using arterial spin labeled perfusion MRI. Neuroimage 59, 2743-2750. doi: 10.1016/j.neuroimage.2011.10.033

Focke, N. K., Helms, G., Scheewe, S., Pantel, P. M., Bachmann, C. G., Dechent, P., et al. (2011). Individual voxel-based subtype prediction can differentiate progressive supranuclear palsy from idiopathic Parkinson syndrome and healthy controls. Hum. Brain Mapp. 32, 1905-1915. doi: 10.1002/hbm.21161

Forstmann, B. U., Van Den Wildenberg, W. P., and Ridderinkhof, K. R. (2008). Neural mechanisms, temporal dynamics, and individual differences in interference control. J. Cogn. Neurosci. 20, 1854-1865. doi: 10.1162/jocn.2008.20122

Gelb, D. J., Oliver, E., and Gilman, S. (1999). Diagnostic criteria for Parkinson disease. Arch. Neurol. 56, 33-39. doi: 10.1001/archneur.56.1.33

Ghosh, B. C., Calder, A. J., Peers, P. V., Lawrence, A. D., Acosta-Cabronero, J., Pereira, J. M., et al. (2012). Social cognitive deficits and their neural correlates in progressive supranuclear palsy. Brain 135, 2089-2102. doi: 10.1093/brain/aws128

Giordano, A., Tessitore, A., Corbo, D., Cirillo, G., De Micco, R., Russo, A., et al. (2013). Clinical and cognitive correlations of regional gray matter atrophy in progressive supranuclear palsy. Parkinsonism Relat. Disord. 19, 590-594. doi: 10.1016/j.parkreldis.2013.02.005

Halliday, G. M. (2009). Thalamic changes in Parkinson's disease. Parkinsonism Relat. Disord. 15 (Suppl. 3), S152-S155. doi: 10.1016/S1353-8020(09)70804-1
Halliday, G. M., McRitchie, D. A., Cartwright, H., Pamphlett, R., Hely, M. A., and Morris, J. G. (1996). Midbrain neuropathology in idiopathic Parkinson's disease and diffuse Lewy body disease. J. Clin. Neurosci. 3, 52-60. doi: 10.1016/S09675868(96)90083-1

Herrero, M. T., Barcia, C., and Navarro, J. M. (2002). Functional anatomy of thalamus and basal ganglia. Childs Nerv. Syst. 18, 386-404. doi: 10.1007/s00381002-0604-1

Hirano, S., Shinotoh, H., Shimada, H., Aotsuka, A., Tanaka, N., Ota, T., et al. (2010). Cholinergic imaging in corticobasal syndrome, progressive supranuclear palsy and frontotemporal dementia. Brain 133, 2058-2068. doi: 10.1093/brain/awq120

Hong, J. Y., Lee, J. E., Sohn, Y. H., and Lee, P. H. (2012). Neurocognitive and atrophic patterns in Parkinson's disease based on subjective memory complaints. J. Neurol. 259, 1706-1712. doi: 10.1007/s00415-011-6404-3

Hughes, A. J., Daniel, S. E., Kilford, L., and Lees, A. J. (1992). Accuracy of clinical diagnosis of idiopathic Parkinson's disease: a clinico-pathological study of 100 cases. J. Neurol. Neurosurg. Psychiatry 55, 181-184. doi: 10.1136/jnnp.55.3.181

Ibarretxe-Bilbao, N., Junque, C., Segura, B., Baggio, H. C., Marti, M. J., Valldeoriola, F., et al. (2012). Progression of cortical thinning in early Parkinson's disease. Mov. Disord. 27, 1746-1753. doi: 10.1002/mds.25240

Jubault, T., Brambati, S. M., Degroot, C., Kullmann, B., Strafella, A. P., Lafontaine, A. L., et al. (2009). Regional brain stem atrophy in idiopathic Parkinson's disease detected by anatomical MRI. PLOS ONE 4:e8247. doi: 10.1371/journal.pone.0008247

Juh, R., Kim, J., Moon, D., Choe, B., and Suh, T. (2004). Different metabolic patterns analysis of Parkinsonism on the 18F-FDG PET. Eur. J. Radiol. 51, 223-233. doi: 10.1016/S0720-048X(03)00214-6

Juh, R., Pae, C. U., Kim, T. S., Lee, C. U., Choe, B., and Suh, T. (2005). Cerebral glucose metabolism in corticobasal degeneration comparison with progressive supranuclear palsy using statistical mapping analysis. Neurosci. Lett. 383, 22-27. doi: 10.1016/j.neulet.2005.03.057

Karagulle Kendi, A. T., Lehericy, S., Luciana, M., Ugurbil, K., and Tuite, P. (2008). Altered diffusion in the frontal lobe in Parkinson disease. AJNR Am. J. Neuroradiol. 29, 501-505. doi: 10.3174/ajnr.A0850

Kato, N., Arai, K., and Hattori, T. (2003). Study of the rostral midbrain atrophy in progressive supranuclear palsy. J. Neurol. Sci. 210, 57-60. doi: 10.1016/S0022510X(03)00014-5

Kehagia, A. A., Barker, R. A., and Robbins, T. W. (2012). Revisiting the effects of Parkinson's disease and frontal lobe lesions on task switching: the role of rule reconfiguration. J. Neuropsychol. doi: 10.1111/jnp.12004. [Epub ahead of print].

Kikuchi, A., Takeda, A., Kimpara, T., Nakagawa, M., Kawashima, R., Sugiura, M., et al. (2001). Hypoperfusion in the supplementary motor area, dorsolateral prefrontal cortex and insular cortex in Parkinson's disease. J. Neurol. Sci. 193, 29-36. doi: 10.1016/S0022-510X(01)00641-4

Kimura, N., Hanaki, S., Masuda, T., Hanaoka, T., Hazama, Y., Okazaki, T., et al. (2011). Brain perfusion differences in parkinsonian disorders. Mov. Disord. 26, 2530-2537. doi: 10.1002/mds.23915

Kostic, V. S., Agosta, F., Petrovic, I., Galantucci, S., Spica, V., JecmenicaLukic, M., et al. (2010). Regional patterns of brain tissue loss associated with depression in Parkinson disease. Neurology 75, 857-863. doi: 10.1212/WNL.0b013e3181f11cld

Laird, A. R., Fox, P. M., Price, C. J., Glahn, D. C., Uecker, A. M., Lancaster, J. L., et al. (2005). ALE meta-analysis: controlling the false discovery rate and performing statistical contrasts. Hum. Brain Mapp. 25, 155-164. doi: 10.1002/ hbm. 20136

Lancaster, J. L., Tordesillas-Gutierrez, D., Martinez, M., Salinas, F., Evans, A., Zilles, K., et al. (2007). Bias between MNI and Talairach coordinates analyzed using the ICBM-152 brain template. Hum. Brain Mapp. 28, 1194-1205. doi: 10.1002/hbm.20345

Larsen, J. P., Dupont, E., and Tandberg, E. (1994). Clinical diagnosis of Parkinson's disease. Proposal of diagnostic subgroups classified at different levels of confidence. Acta. Neurol. Scand. 89, 242-251. doi: 10.1111/j.1600-0404.1994. tb01674.x

Lee, J. E., Park, H. J., Song, S. K., Sohn, Y. H., Lee, J. D., and Lee, P. H. (2010). Neuroanatomic basis of amnestic MCI differs in patients with and without Parkinson disease. Neurology 75, 2009-2016. doi: 10.1212/WNL.0b013e3181ff96bf

Lehericy, S., Hartmann, A., Lannuzel, A., Galanaud, D., Delmaire, C., Bienaimee, M. J., et al. (2010). Magnetic resonance imaging lesion pattern in Guadeloupean 
parkinsonism is distinct from progressive supranuclear palsy. Brain 133, 2410-2425. doi: 10.1093/brain/awq162

Martin, W. R., Wieler, M., Gee, M., and Camicioli, R. (2009). Temporal lobe changes in early, untreated Parkinson's disease. Mov. Disord. 24, 1949-1954. doi: $10.1002 / \mathrm{mds} .22680$

Meppelink, A. M., De Jong, B. M., Teune, L. K., and Van Laar, T. (2011). Regional cortical grey matter loss in Parkinson's disease without dementia is independent from visual hallucinations. Mov. Disord. 26, 142-147. doi: 10.1002/mds.23375

Nagano-Saito, A., Washimi, Y., Arahata, Y., Kachi, T., Lerch, J. P., Evans, A. C., et al. (2005). Cerebral atrophy and its relation to cognitive impairment in Parkinson disease. Neurology 64, 224-229. doi: 10.1212/01.WNL.0000149510.41793.50

Nath, U., Ben-Shlomo, Y., Thomson, R. G., Morris, H. R., Wood, N. W., Lees, A. J., et al. (2001). The prevalence of progressive supranuclear palsy (SteeleRichardson-Olszewski syndrome) in the UK. Brain 124, 1438-1449. doi: 10.1093/brain/124.7.1438

Nilsson, C., Markenroth Bloch, K., Brockstedt, S., Latt, J., Widner, H., and Larsson, E. M. (2007). Tracking the neurodegeneration of parkinsonian disorders-a pilot study. Neuroradiology 49, 111-119. doi: 10.1007/s00234-006-0165-1

Padovani, A., Borroni, B., Brambati, S. M., Agosti, C., Broli, M., Alonso, R., et al. (2006). Diffusion tensor imaging and voxel based morphometry study in early progressive supranuclear palsy. J. Neurol. Neurosurg. Psychiatry 77, 457-463. doi: 10.1136/jnnp.2005.075713

Pan, P. L., Song, W., and Shang, H. F. (2012). Voxel-wise meta-analysis of gray matter abnormalities in idiopathic Parkinson's disease. Eur. J. Neurol. 19, 199-206. doi: 10.1111/j.1468-1331.2011.03474.x

Pavese, N., Metta, V., Bose, S. K., Chaudhuri, K. R., and Brooks, D. J. (2010). Fatigue in Parkinson's disease is linked to striatal and limbic serotonergic dysfunction. Brain 133, 3434-3443. doi: 10.1093/brain/awq268

Pereira, J. B., Junque, C., Marti, M. J., Ramirez-Ruiz, B., Bargallo, N., and Tolosa, E. (2009). Neuroanatomical substrate of visuospatial and visuoperceptual impairment in Parkinson's disease. Mov. Disord. 24, 1193-1199. doi: $10.1002 / \mathrm{mds} .22560$

Picton, T. W., Stuss, D. T., Alexander, M. P., Shallice, T., Binns, M. A., and Gillingham, S. (2007). Effects of focal frontal lesions on response inhibition. Cereb. Cortex 17, 826-838. doi: 10.1093/cercor/bhk031

Pinto, S., Thobois, S., Costes, N., Le Bars, D., Benabid, A. L., Broussolle, E., et al. (2004). Subthalamic nucleus stimulation and dysarthria in Parkinson's disease: a PET study. Brain 127, 602-615. doi: 10.1093/brain/awh074

Ramirez-Ruiz, B., Marti, M. J., Tolosa, E., Gimenez, M., Bargallo, N., Valldeoriola, F., et al. (2007). Cerebral atrophy in Parkinson's disease patients with visual hallucinations. Eur. J. Neurol. 14, 750-756. doi: 10.1111/j.1468-1331.2007.01768.x

Rizzolatti, G., Fogassi, L., and Gallese, V. (2002). Motor and cognitive functions of the ventral premotor cortex. Curr. Opin. Neurobiol. 12, 149-154. doi: 10.1016/S0959-4388(02)00308-2

Sanchez-Castaneda, C., Rene, R., Ramirez-Ruiz, B., Campdelacreu, J., Gascon, J., Falcon, C., et al. (2009). Correlations between gray matter reductions and cognitive deficits in dementia with Lewy Bodies and Parkinson's disease with dementia. Mov. Disord. 24, 1740-1746. doi: 10.1002/mds.22488

Stamelou, M., De Silva, R., Arias-Carrion, O., Boura, E., Hollerhage, M., Oertel, W. H., et al. (2010). Rational therapeutic approaches to progressive supranuclear palsy. Brain 133, 1578-1590. doi: 10.1093/brain/awq115

Stefurak, T., Mikulis, D., Mayberg, H., Lang, A. E., Hevenor, S., Pahapill, P., et al. (2003). Deep brain stimulation for Parkinson's disease dissociates mood and motor circuits: a functional MRI case study. Mov. Disord. 18, 1508-1516. doi: 10.1002/mds. 10593

Stroup, D. F., Berlin, J. A., Morton, S. C., Olkin, I., Williamson, G. D., Rennie, D., et al. (2000). Meta-analysis of observational studies in epidemiology: a proposal for reporting. Meta-analysis Of Observational Studies in Epidemiology (MOOSE) group. JAMA 283, 2008-2012. doi: 10.1001/jama.283.15.2008
Takahashi, R., Ishii, K., Kakigi, T., Yokoyama, K., Mori, E., and Murakami, T. (2011). Brain alterations and mini-mental state examination in patients with progressive supranuclear palsy: voxel-based investigations using ffluorodeoxyglucose positron emission tomography and magnetic resonance imaging. Dement. Geriatr. Cogn. Dis. Extra 1, 381-392. doi: 10.1159/000333368

Tanner, C. M., and Goldman, S. M. (1996). Epidemiology of Parkinson's disease. Neurol. Clin. 14, 317-335. doi: 10.1016/S0733-8619(05)70259-0

Tessitore, A., Esposito, F., Vitale, C., Santangelo, G., Amboni, M., Russo, A., et al. (2012). Default-mode network connectivity in cognitively unimpaired patients with Parkinson disease. Neurology 79, 2226-2232. doi: 10.1212/WNL.0b013e31827689d6

Tir, M., Delmaire, C., Le Thuc, V., Duhamel, A., Destee, A., Pruvo, J. P., et al. (2009). Motor-related circuit dysfunction in MSA-P: usefulness of combined whole-brain imaging analysis. Mov. Disord. 24, 863-870. doi: 10.1002/mds. 22463

Turkeltaub, P. E., Eden, G. F., Jones, K. M., and Zeffiro, T. A. (2002). Meta-analysis of the functional neuroanatomy of single-word reading: method and validation. Neuroimage 16, 765-780. doi: 10.1006/nimg.2002.1131

Varrone, A., Pagani, M., Salvatore, E., Salmaso, D., Sansone, V., Amboni, M., et al. (2007). Identification by [99mTc]ECD SPECT of anterior cingulate hypoperfusion in progressive supranuclear palsy, in comparison with Parkinson's disease. Eur. J. Nucl. Med. Mol. Imaging 34, 1071-1081. doi: 10.1007/s00259-0060344-7

Whitwell, J. L., Avula, R., Master, A., Vemuri, P., Senjem, M. L., Jones, D. T., et al. (2011). Disrupted thalamocortical connectivity in PSP: a resting-state fMRI, DTI, and VBM study. Parkinsonism Relat. Disord. 17, 599-605. doi: 10.1016/j.parkreldis.2011.05.013

Williams, D. R., De Silva, R., Paviour, D. C., Pittman, A., Watt, H. C., Kilford, L., et al. (2005). Characteristics of two distinct clinical phenotypes in pathologically proven progressive supranuclear palsy: richardson's syndrome and PSP-parkinsonism. Brain 128, 1247-1258. doi: 10.1093/brain/awh488

Williams, D. R., and Lees, A. J. (2009). Progressive supranuclear palsy: clinicopathological concepts and diagnostic challenges. Lancet Neurol. 8, 270-279. doi: 10.1016/S1474-4422(09)70042-0

Yang, H., Zhou, X. J., Zhang, M. M., Zheng, X. N., Zhao, Y. L., and Wang, J. (2013). Changes in spontaneous brain activity in early Parkinson's disease. Neurosci. Lett. 549, 24-28. doi: 10.1016/j.neulet.2013.05.080

Zwergal, A., La Fougere, C., Lorenzl, S., Rominger, A., Xiong, G., Deutschenbaur, L., et al. (2011). Postural imbalance and falls in PSP correlate with functional pathology of the thalamus. Neurology 77, 101-109. doi: 10.1212/WNL.0b013e318223c79d

Conflict of Interest Statement: The authors declare that the research was conducted in the absence of any commercial or financial relationships that could be construed as a potential conflict of interest.

Received: 29 November 2013; accepted: 26 January 2014; published online: 18 February 2014.

Citation: Shao N, Yang J, Li J and Shang H-F (2014) Voxelwise meta-analysis of gray matter anomalies in progressive supranuclear palsy and Parkinson's disease using anatomic likelihood estimation. Front. Hum. Neurosci. 8:63. doi: 10.3389/fnhum. 2014.00063

This article was submitted to the journal Frontiers in Human Neuroscience.

Copyright (C) 2014 Shao, Yang, Li and Shang. This is an open-access article distributed under the terms of the Creative Commons Attribution License (CC BY). The use, distribution or reproduction in other forums is permitted, provided the original author(s) or licensor are credited and that the original publication in this journal is cited, in accordance with accepted academic practice. No use, distribution or reproduction is permitted which does not comply with these terms. 